\title{
QUEL RECOURS À L'APPRENTISSAGE EN ITALIE ? RETOUR SUR LES RÉFORMES RÉCENTES ET LEUR SAISIE PAR LES ACTEURS
}

\author{
Maël Dif-Pradalier et Samuel Zarka
}

Céreq | « Formation emploi »

2019/3 n 147 | pages 69 à 88

ISSN 0759-6340

Article disponible en ligne à l'adresse :

https://www.cairn.info/revue-formation-emploi-2019-3-page-69.htm

Distribution électronique Cairn.info pour Céreq.

(C) Céreq. Tous droits réservés pour tous pays.

La reproduction ou représentation de cet article, notamment par photocopie, n'est autorisée que dans les limites des conditions générales d'utilisation du site ou, le cas échéant, des conditions générales de la licence souscrite par votre établissement. Toute autre reproduction ou représentation, en tout ou partie, sous quelque forme et de quelque manière que ce soit, est interdite sauf accord préalable et écrit de l'éditeur, en dehors des cas prévus par la législation en vigueur en France. Il est précisé que son stockage dans une base de données est également interdit. 
FORMATION Formation emploi

Revue française de sciences sociales

147 | Juillet-Septembre

Italie : réformes politiques et permanences sociétales des relations formation-emploi

\section{Quel recours à l'apprentissage en Italie ? Retour sur les réformes récentes et leur saisie par les acteurs}

How to resort to apprenticeship in Italy? A review of recent reforms and how it has been adopted by the actors

Ein Rückblick auf die neusten Reformen und deren Akzeptanz unter den

Akteuren

¿Cómo se recurre al aprendizaje en Italia? Retorno sobre las reformas recientes

y su captura por parte de los actores

Maël Dif-Pradalier et Samuel Zarka

\section{OpenEdition}

\section{Journals}

Édition électronique

URL : http://journals.openedition.org/formationemploi/7612

ISSN : 2107-0946

Éditeur

La Documentation française

Édition imprimée

Date de publication : 31 octobre 2019

Pagination : 69-88

ISSN : 0759-6340

Distribution électronique Cairn

\section{CAIRN INFO}

CHERCHER, REPÉRER, AVANCER

Référence électronique

Maël Dif-Pradalier et Samuel Zarka, «Quel recours à l'apprentissage en Italie? Retour sur les réformes récentes et leur saisie par les acteurs », Formation emploi [En ligne], 147 | Juillet-Septembre, mis en ligne le 03 janvier 2021, consulté le 31 octobre 2019. URL : http://journals.openedition.org/ formationemploi/7612 


\title{
Quel recours à l'apprentissage en Italie? Retour sur les réformes récentes et leur saisie par les acteurs
}

\author{
MaËL Dif-Pradalier \\ Sociologue, Professeur, HES-SO, Haute Ecole de travail social de Fribourg (Suisse)
}

SAMUEL ZaRKA

Doctorant en sociologie, attaché temporaire d'enseignement et de recherche, Laboratoire interdisciplinaire pour la sociologie économique, Conservatoire National des Arts et

Métiers, France

Résumé

Quel recours à l'apprentissage en Italie ? Retour sur les réformes récentes et leur saisie par les acteurs

Le manque d'engagement des entreprises italiennes dans l'apprentissage est fréquemment corrélé au coût du dispositif, comparativement à d'autres formules contractuelles. Toutefois, ce résultat interroge : si la concurrence entre contrats est si vive, comment saisir le recours à l'apprentissage lorsqu'il a lieu ? Pour répondre à cette question, l'article part de textes règlementaires et de statistiques touchant le fait apprenti en Italie, qui seront mis en regard d'un matériau d'entretiens auprès d'acteurs de l'apprentissage (responsables régionaux, employeurs et apprentis). Cette démarche permet de réveler plusieurs types d'encastrement au sein desquels le contrat d'apprentissage peut prendre sens : les compromis d'une entreprise familiale du nord du pays ou la perspective d'émigration d'un jeune diplômé d'un institut technique. De ce fait, cet article nous renseigne sur les conditions de primo-engagement en Italie.

Mots clés : apprentissage, législation de l'apprentissage, stage de formation, disparité régionale, Italie

1. Les auteurs remercient les coordinateurs de ce numéro spécial de Formation Emploi, l'équipe de rédaction, ainsi que les lecteurs anonymes de la revue pour les relectures attentives et constructives qu'ils ont bien voulu faire de ce texte. 
Abstract

How to resort to apprenticeship in Italy? A review of recent reforms and how it has been adopted by the actors

The lack of commitment of Italian companies in apprenticeship is frequently correlated to its relatively high cost compared to other contractual possibilities. However, one could challenge this oft-invoked explanation: if the competition between employment contracts is so intense, how can one understand resorting to apprenticeship when it takes place? To address this question, this article starts from an analysis of regulatory texts and statistics concerning apprenticeship in Italy and confronts it with interviews conducted with actors of apprenticeship (regional managers, employers and apprentices). This approach reveals several types of embeddedness in which apprenticeship contracts can make sense: within the compromises of a family business in the North of the country or through the prospect of emigration of a young graduate of a technical institute. Consequently, this article illustrates conditions under which firsttime entrants access the labour market.

Keywords: apprenticeship, apprenticeship legislation, traineeship, regional disparity, Italy

Journal of Economic Literature : M 53

Traduction : auteurs.

\section{Introduction}

En Italie, la formation par apprentissage, faisant alterner périodes de travail en entreprise et périodes de formation en école, est fréquemment convoquée par les représentants politiques, de droite comme de gauche, pour justifier le bien-fondé d'une inflexion significative de la politique éducative (par ex. Bobba, 2015) ${ }^{2}$. Parce qu'ils seraient formés au plus près des réalités productives, les jeunes passés par l'apprentissage répondraient mieux aux attentes des employeurs que les jeunes issus de formation plus scolaires. Ils seraient de ce fait plus employables et moins touchés par le chômage.

En 2012, la réforme Fornero ${ }^{3}$, en occasionnant une refonte de l'apprentissage, a constitué un moment fort de cette rhétorique. Or, malgré une reprise relative en 2016

2. Cette doctrine, qui prend régulièrement pour exemple l'Allemagne ou la Suisse, est relayée par des organisations telles que le CEDEFOP (Centre européen pour le développement de la formation professionnelle) et l'OCDE (Organisation de coopération et de développement économiques), qui ont invité l'Italie à améliorer la qualité de son système d'apprentissage encore récemment (CEDEFOP, 2017 ; OECD, 2017a et b). En Italie, l'Agence nationale des politiques actives de l'emploi (Agencia Nazionale Politiche Attive del Lavoro), dépendant du ministère du Travail, assure aussi une promotion continue de l'apprentissage.

3. Du nom de la ministre du Travail du gouvernement de Mario Monti, Président du Conseil. Ce gouvernement « technique » est resté au pouvoir du 16 novembre 2011 au 28 avril 2013. La réforme du marché du 
(INPS et INAPP, 2017), le nombre d'apprentis italiens n'a cessé de baisser depuis 2010, que ce soit par rapport à la population en formation ou à la population active. Pour cette raison même, la réforme Fornero apparaît comme un moment-clé pour apprécier les usages et limites du dispositif en Italie, qui n’a été modifié qu'à la marge ultérieurement ${ }^{4}$.

Le constat d'un manque d'engagement des entreprises italiennes dans l'apprentissage est partagé par plusieurs études récentes (par ex. Cedefop, op. cit. ; INPS et INAPP, op. cit.. ; Muehlemann \& al., 2018). Pour l'expliquer, Muehlemann \& al. (ibid.) utilisent un modèle coûts-bénéfices, déjà appliqué au cas suisse (par ex. Wolter \& Schweri, 2002) : ils mettent en avant le coût du dispositif pour les entreprises, comparativement à d'autres formules contractuelles plus souples et surtout moins onéreuses. La concurrence entre différents types de contrats jouerait donc un rôle central dans le non-recours à l'apprentissage. De fait, il faut souligner le très grand nombre de types de contrats de travail existant en Italie, plus d'une quarantaine en tout.

Cette dimension économique déjouerait l'horizon normatif mettant en valeur l'apprentissage comme support de formation. Ce résultat induit toutefois d'autres questions : comment saisir le recours à l'apprentissage malgré tout ? Des facteurs contextuels le favorisent-ils ? Le volet formation est-il dès lors assumé par les employeurs ?

Considérant par hypothèse que la concurrence entre contrats n'épuise pas la question de l'embauche d'apprentis en Italie, on adoptera une démarche complémentaire aux études pré-citées, visant à comprendre les problématiques de primo-engagement et d'insertion des apprentis italiens. À cette fin, une lecture des statistiques touchant le fait apprenti sera mise en regard d'un matériau d'entretiens avec plusieurs de ses acteurs directs : responsables régionaux, représentants syndicaux, employeurs et apprentis. Croiser les niveaux (macro et micro) et les échelles (nationale, régionale et individuelle) doit permettre de restituer des pratiques à la fois situées et significatives de l'apprentissage en Italie, au-delà de ce qu'une approche économique ne permet, au mieux, que de suggérer.

Cet article procède en trois temps. Premièrement, il rappelle l'échec quantitatif du développement de l'apprentissage en Italie ${ }^{5}$, malgré les évolutions réglementaires et

travail, mise en œuvre par la loi n ${ }^{\circ} 92$ du 28 juin 2012, s’inscrit dans la feuille de route définie par la Banque centrale européenne, dans la lettre du 5 août 2011 adressée au président du Conseil d'alors, Silvio Berlusconi, et qui prévoyait de libéraliser les services et les professions, de privatiser les services publics locaux, de réformer le système des retraites, de baisser les salaires des fonctionnaires et de réformer le marché du travail. 4. Les réformes post-Fornero ne situent plus l'apprentissage au centre d'un ensemble coordonné d'interventions, mais se contentent de modifications réglementaires peu articulées entre elles et sources d'instabilité juridique (Tiraboschi, 2015).

5. On constate des défis similaires auxquels sont confrontées des politiques de développement de l'apprentissage en France, mais aussi en Allemagne (Mona Granato \& Gilles Moreau, «Introduction : les défis de 
normatives engagées par la réforme Fornero. Puis il analyse l'inertie du fait apprenti, en fonction de facteurs contextuels décelables au point de vue statistique et saillants dans les entretiens. Enfin, il présente deux expériences d'apprentis et d'employeurs italiens, révélatrices de l'inscription sociale de ce type de formation, qu'il s'agisse de l'économie familiale ou d'une perspective d'émigration.

\section{Encadré 1. Méthodologie}

Cet article est issu de deux études. La première a été réalisée dans le cadre de l'agence d'objectifs de l'IRES (Institut de recherches économiques et sociales), en 2012 et 2013 (Dif-Pradalier et Zarka, 2014) et propose une comparaison de l'apprentissage en Italie, en France et en Suisse. Cette étude s'appuie principalement sur une enquête qualitative par entretiens semi-directifs : six en Lombardie (Italie); douze en lle-de-France (France); cinq dans le Canton de Vaud (Suisse). Elle a porté aussi bien sur des secteurs traditionnels de l'apprentissage (comme la métallurgie) que sur des secteurs plus récents (services à la personne).

La seconde étude, complémentaire, a été financée par le Fonds national suisse de la recherche scientifique (FNS), entre décembre 2014 et août $2016\left(^{*}\right)$. Elle porte sur le volet italien d'une recherche sur l'apprentissage au sein de groupements ou réseaux d'entreprises formatrices dans les mêmes pays que la première étude. Cette enquête a permis de réaliser dix entretiens complémentaires, en Italie, auprès d'apprentis, de représentants d'entreprises formatrices et de centres de formation, de responsables administratifs, de représentants syndicaux et d'employeurs du secteur de l'industrie.

Dans les deux enquêtes, le choix des personnes interrogées est similaire : il a visé à remonter une chaîne d'intervenants, en partant de l'expérience de formation (entretiens avec des apprentis, formateurs, employeurs) vers différents acteurs institutionnels directement impliqués dans la gestion de l'apprentissage (responsables de centres d'apprentissage, administrateurs régionaux, responsables syndicaux au niveau de fédérations). Dans les deux enquêtes, le matériau de première main est complété par une revue documentaire consacrée à l'expérience d'apprentis, par la lecture de littérature grise et spécialisée, enfin, par l'exploitation de données statistiques.

Dans le présent article, on recourra aux entretiens menés en Italie. Les questions ont porté sur le choix du recours à l'apprentissage, l'expérience de la formation, son organisation, les objectifs poursuivis par sa promotion, enfin, le parcours biographique des apprentis.

(*) Dif-Pradalier M., « L'apprentissage dans le cadre d'un réseau ou groupement d'entreprises en Suisse, en France et en Italie. Vers un nouveau compromis social dans la relation d'emploi ? ", bourse de recherche "Advanced Postdoc.Mobility", n P300P1_151177.

l'apprentissage en Allemagne ", Formation Emploi [Online], 146 | Avril-Juin 2019, Online since 26 June 2019, connection on 15 July 2019. URL : http://journals.openedition.org/formationemploi/7326 


\section{La réforme de l'apprentissage en Italie : un échec quantitatif}

Dans cette partie, on revient sur l'évolution règlementaire de l'apprentissage portée par la réforme Fornero (1.1), avant d'envisager les effets de cette réforme sur le nombre et la répartition des apprentis italiens (1.2).

\subsection{Réforme Fornero : l'apprentissage comme « voie d'entrée principale des jeunes sur le marché du travail »}

En Italie, l'apprentissage est initialement codifié dans la loi de 1955, qui prévoit que la formation professionnelle de l'apprenti doit se partager entre formation pratique (on the job) et enseignement complémentaire (obligatoire et gratuit) ${ }^{6}$. Suite au "Paquet Treu " (1997), puis à la réforme Biagi (2003) 7 , la réglementation de l'apprentissage a connu une forte évolution avec la réforme Fornero de 2012. Cette réforme devait prendre le contrepied de la baisse des effectifs au cours des années précédentes ${ }^{8}$. Elle reprend à son compte l'essentiel des dispositions consignées quelques mois plus tôt, dans un document intitulé "Texte unique sur l'apprentissage " (Testo unico dell'apprendistato), résultat d'un accord entre la principale organisation patronale italienne (Confindustria) et les trois confédérations syndicales (Confederagione generale italiana del lavoro - CGIL, Confederazione Italiana Sindacato Lavoratori - CISL et Unione Italiana del Lavoro - UIL).

6. Selon la loi de 1955, l’apprentissage est un contrat à durée indéterminée, dont seule la période de formation est limitée.

7. En Italie, la promotion de l'apprentissage commence dans les années 1990. Le « Paquet Treu (1997) aménage la loi de 1955. L'âge maximum d'accès à l'apprentissage passe de 24 à 26 ans et la durée du contrat peut dorénavant varier de 18 mois à 4 ans (et non plus cinq ans comme auparavant, à l'exception du secteur de l'artisanat). Les entreprises sont incitées à engager des jeunes par des allégements de " charges sociales" sur les salaires des apprentis. Ces derniers doivent en principe participer à une formation hors de l'entreprise. En entreprise, ils sont encadrés par un tuteur (tutor), qui doit désormais justifier d'au moins trois années d'expérience professionnelle et ne peut encadrer plus de cinq apprentis à la fois. En 2003, la réforme Biagi vise à flexibiliser les canaux d'entrée en emploi, à défaut d'assouplir les conditions d'interruption de la relation de travail.

8. Passé de 646000 en 2008 à moins de 542000 en 2010, soit une chute de près de neuf points sur la période (ISFOL, 2011 et 2012). 
Dans son article 1er, la loi de 2012 reprend l'objectif du Testo unico : "Valoriser l'apprentissage comme modalité principale d'entrée des jeunes dans le monde du travail ». Elle confirme également les trois types de contrats existant depuis la réforme Biagi, puis définis dans l'article 2 du Testo unico:

Type 1 : l'apprentissage pour la qualification et la certification professionnelle (per la qualifica e per il diploma professionale ${ }^{10}$ ) : il concerne les jeunes âgés de 15 à 24 ans, dure au maximum 3 ans ${ }^{11}$, comprend 400 heures de formation hors de l'entreprise et vise l'obtention d'un diplôme. Il est pensé comme un instrument de lutte contre les sorties précoces du système scolaire (drop-outs). C'est le type de contrat qui se rapproche le plus de l'apprentissage dual promu au niveau international (par ex. Cedefop, op. cit., p. 15).

Type 2 : l'apprentissage professionnel ou de métier (professionalizzante o contratto di mestiere $^{12}$ ) : il s'adresse aux jeunes de 18 à 29 ans, ne peut durer plus de trois ans et réduit la formation hors de l'entreprise à 120 heures sur trois ans (contre 120 heures par an auparavant). Il est mis en œuvre et réglementé par les régions, en concertation avec les partenaires sociaux. Il doit permettre aux apprentis d'acquérir les compétences nécessaires à l'exercice d'un métier déterminé, selon les conditions définies dans une convention collective. Ce type d'apprentissage concentre les effectifs les plus nombreux.

Type 3 : l'apprentissage de formation supérieure et de recherche (di alta formazione e ricerca) : il vise à la fois l'obtention d'un diplôme de deuxième ou troisième cycle universitaire et à favoriser l'insertion professionnelle des jeunes chercheurs dans les entreprises. Sa durée varie en fonction du diplôme préparé et sa mise en ouvre dépend aussi des régions, tout en faisant appel à un partenariat avec les universités.

La réforme Fornero clarifie les règles en matière de pilotage de l'apprentissage, jusquelà sources de conflits entre le niveau national et le niveau régional, et, partant, d'immobilisme (Facello, 2013). Le gouvernement a désormais en charge la fixation des grandes lignes du contrat d'apprentissage, tandis que les partenaires sociaux déterminent les modalités de formation et que les régions assurent le suivi en entreprise. L'introduction de ce système de gouvernance croisée entre État, régions et partenaires sociaux serait l'élément le plus novateur de la réforme, d'après certains commentateurs (Teoldi \& Garibaldi, 2012 ; Pastore, 2014 ; Cefalo, 2015) et répond à son objectif affirmé de promotion du dispositif.

9. "Valorizzando l'apprendistato comme modalità prevalente di ingresso dei giovani nel mondo del lavoro ".

10. Avec le "Jobs Act» de 2015 (loi 81/2015), son appellation devient "l'apprendistato per la qualifica e il diploma professionale, il diploma di istruzione secondaria superiore e il certificato di specializzazione tecnica superiore".

11. Sauf si la durée du diploma professionale régional est de 4 ans.

12. Avec le "Jobs Act", la mention " contratto di mestiere " disparaît. 
Pour les trois types d'apprentissage, l'employeur bénéficie d'importantes exonérations de cotisations sociales et de déductions fiscales sur toute la durée du contrat. En échange, il doit mettre en place un plan de formation individuel (piano formativo individuale), rapporter et consigner les compétences acquises par l'apprenti dans un livret de formation (libretto formativo). L'apprenti peut être engagé jusqu'à deux niveaux en dessous de la qualification à laquelle il est censé accéder à l'issue de sa formation. La fixation de la rétribution en fonction de sa productivité est interdite (divieto di retribuzione a cottimo ; interdiction du salaire à la pièce) ${ }^{13}$. La durée minimale d'exercice professionnel est de six mois, afin de prémunir le dévoiement de l'apprentissage. L'annulation (recesso) du contrat est interdite, sauf en cas de juste cause ou de motif justifié (giusta causa o giustificato motivo) avancé par l'une des deux parties. Enfin, la réforme impose un contrat écrit et prévoit des sanctions financières en cas de nonrespect des engagements de la part de l'employeur.

$\mathrm{Au}$ sein de ce cadrage règlementaire, l'apprentissage revêt deux volets : formation et emploi (occupazione).

Du point de vue de la formation, l'obligation est reconduite, pour chaque apprenti, d'être accompagné dans l'entreprise par un tuteur (tutor), qui doit être formé. En Lombardie, par exemple, la formation des formateurs, financée par la région, est gratuite pour les employeurs ${ }^{14}$. Elle se déroule généralement dans un Centro formativo provinciale (CFP). Toutefois, ces centres sont très peu contrôlés en termes de contenus et de qualité de la formation professionnelle qu'ils dispensent.

Quant au volet emploi, il traduit la volonté politique d'utiliser l'apprentissage comme un levier permettant à la fois d'augmenter le nombre de jeunes en emploi et de diminuer le nombre de dropout et de NEET (Not in education, employment, or training ${ }^{15}$ ). Ainsi, la loi augmente le nombre maximum d'apprentis qu'il est possible d'engager en fonction du personnel qualifié présent dans l'entreprise ${ }^{16}$. De plus, dans le cas où l'employeur souhaiterait embaucher de nouveaux apprentis, il doit maintenir le lien

13. La fixation de la rémunération est laissée à la négociation collective, qui a le choix entre deux formules : 1) l'engagement de l'apprenti à un niveau de qualification pouvant descendre jusqu'à deux niveaux au-dessous du poste occupé, ou bien 2) un pourcentage du salaire des professionnels qualifiés, effectuant l'ensemble des tâches et missions que l'apprenti devra accomplir.

14. Elle est payante et accélérée en cas de délais d'attente trop longs ou d'urgence de la part des employeurs, en raison des sanctions financières importantes relatives au non-respect de l'obligation de formation du tuteur (entretien avec la responsable d'un des CFP de la province de Brescia).

15. Décrocheur, sans emploi et ne suivant ni enseignement ni formation.

16. La réforme Fornero a confirmé le rapport, déjà prévu dans le "Texte unique ", de un à un entre les apprentis et les travailleurs qualifiés en interne, dans le cas des entreprises de moins de dix salariés. Pour les autres cas, le rapport est de trois apprentis pour deux salariés qualifiés. Si l'entreprise ne compte aucun salarié ou si le nombre de salariés est inférieur à trois, il est possible d'engager jusqu'à trois apprentis. Ces dispositions ne s'appliquent pas au secteur de l'artisanat (Confcommercio, op. cit., p. 23). 
d'emploi pour la moitié ${ }^{17}$ au moins des apprentis déjà dans l'entreprise depuis trois ans. Une fois l'apprentissage achevé, les exonérations de cotisations sociales se poursuivent la première année d'emploi ${ }^{18}$. Enfin, en cas d'absence prouvée de tout contenu de formation, le contrat d'apprentissage est requalifié en contrat de travail à durée indéterminée.

Dans un contexte de baisse continue des effectifs apprentis, la réforme Fornero, en mettant en œuvre une politique dite de l'offre, a cherché à relancer l'apprentissage en le rendant plus attractif pour les entreprises. La prégnance du volet emploi, c'est-à-dire le recours à l'apprentissage comme réponse aux difficultés structurelles rencontrées par les jeunes pour accéder à l'emploi et s'y maintenir, a été confirmée par les modifications réglementaires ultérieures. La plus importante d'entre elles, inscrite dans le décret loi $\mathrm{n}^{\circ} 81$ du 15 juin 2015, vise à promouvoir le recours à l'apprentissage de type 1 et 3 , plutôt que de type 2 . Il prévoit en outre une diminution de la rémunération des apprentis ${ }^{19}$ et l'augmentation des incitations financières à l'embauche (exonérations, primes).

\subsection{Un échec quantitatif lisible dans les statistiques italiennes}

Un regard statistique sur le fait apprenti, durant les années suivant immédiatement la réforme Fornero, amène à considérer son échec. Depuis 2009, la diminution du nombre d'apprentis est quasi constante : de 650 000, les effectifs moyens annuels passent à 446000 en 2014 et à 410000 en 2015 (INPS et INAPP, op. cit.). La proportion d'apprentis reste faible, représentant $12,5 \%$ des actifs occupés âgés de 15 à 29 ans en 2016 (INAPP, ibid.) et entre 2 et $3 \%$ de l'ensemble des actifs occupés (Cefalo, op. cit.).

Le dispositif connaît aussi des disparités régionales. Ainsi, sa diffusion est nettement plus forte dans le nord-ouest du pays (INPS et INAPP, op. cit., p. 6), en Lombardie et Vénétie notamment, mais aussi dans la région autonome de Trentin-Haut-Adige, et particulièrement dans la province de Bolzano, frontalière avec l'Autriche et la Suisse, qui présente le taux d'apprentis non majeurs le plus élevé. Toutefois, la baisse du nombre d'apprentis est commune à toutes les régions. Les variations ont été les plus importantes pour l'apprendistato professionalizzante (type 2), qui, rassemblant plus de

17. Ce pourcentage est réduit à $30 \%$ lors des trois premières années d'application de la loi.

18. Et même la seconde année, dans le cas des entreprises de moins de dix salariés.

19. Avec le "Jobs Act», la loi ne prévoit aucune rétribution pour l'apprenti pour les heures de formation réalisées dans un centre de formation. Pour celles effectuées en entreprise, la rétribution est égale à $10 \%$ du salaire négocié (et s'ajoute à ce-dernier qui ne rémunère que les heures correspondant au volet « emploi »du contrat d'alternance réalisé en entreprise). 
$95 \%$ des apprentis totaux, voit ses effectifs diminuer globalement ${ }^{21}$. En revanche, les deux autres types ( 1 et 3 ) rassemblent moins de $5 \%$ des apprentis à eux deux. L'apprentissage "per la qualifica e per il diploma professionale" (type 1), le plus proche de l'apprentissage dual, n’a jamais "décollé » (autour de $3 \%$ du total des apprentis et est en baisse en $2016^{22}$ ), l'essentiel des contrats de ce type se concentrant au nord-est, zone la plus industrialisée du pays (plus de $40 \%$ encore en 2016). Au total, moins de la moitié des contrats d'apprentissage italiens perdurent au-delà de la première année.

En s'appuyant sur le rapport ISFOL de 2011 (qui porte sur les années 2007-2008), il est possible de fournir un bref aperçu de la situation de l'apprentissage à l'époque de la réforme Fornero. Les apprentis italiens sont avant tout des hommes (58,5\% contre $41,5 \%$ de femmes en 2009) et se répartissent en trois groupes d'âge comparables (32,2\% pour les $18-21$ ans, $32 \%$ pour les $22-24$ ans et $33 \%$ pour les 25 ans et plus ${ }^{23}$.

Tandis que les apprentis hommes appartiennent davantage à la classe d'âge des 18-21 ans, l'âge moyen des femmes apprenties est plus élevé, la majorité d'entre elles ayant plus de 25 ans (ISFOL, 2011, p. 11). L'allongement de la période dite " de jeunesse ", particulièrement net en Italie, se traduit donc dans l'apprentissage, où l'âge moyen des hommes et des femmes augmente (ISFOL, op. cit., p. 11). Cette donnée confirme une caractéristique de ce pays où l'entrée en emploi s'opère tardivement (en 2010, et en moyenne, les Italiens poursuivaient leurs études jusqu'à 26,9 ans ${ }^{24}$ ). En termes de niveau scolaire, 60 \% des apprentis sont titulaires d'un diplôme de niveau baccalauréat (diploma superiore). Seuls $7 \%$ sont titulaires d'un diplôme de l'enseignement supérieur (laurea) ${ }^{25}$.

L'immense majorité des apprentis (93\%) déclare avoir signé un contrat d'apprentissage à défaut d'avoir pu trouver un autre type de contrat, plus protecteur et rémunérateur. La majorité d'entre eux (54\%) déclarait avoir connu des expériences salariées avant l'entrée en apprentissage, tandis que $46 \%$ déclaraient qu'il s'agissait de leur première expérience professionnelle. Un contrat d'apprentissage dure en moyenne 35 mois et chaque apprenti travaille en moyenne 38 heures par semaine chez son

20. 96,5\% selon le XVIIème rapport publié par l'INPS et l'INAPP (op. cit.).

21. Malgré une reprise en 2016 (INPS et INAPP, op. cit., p. 9).

22. 3,3\% en 2015 et 2,8\% en 2016 (INPS et INAPP, op. cit., p 6).

23. Les apprentis appartenant à la classe d'âge 15-17 ans (2,8\%) est résiduelle (ISFOL, op.cit., p. 10).

24. L'âge moyen élevé de poursuite d'études d'une part, la participation relativement faible à l'enseignement supérieur, évoquée plus haut, d'autre part, peuvent apparaître comme une contradiction statistique. En réalité, on peut supposer que l'explication tient au mode d'organisation particulier des études supérieures, en Italie, qui permet notamment de répéter plusieurs fois un même examen jusqu'à l'obtention de la moyenne ou de la note souhaitée, mais aussi, dans certaines disciplines et certaines universités, de fréquenter les cours de l'année supérieure sans avoir validé les cours de l'année précédente.

25. De manière plus générale, l'Italie se caractérise par la plus faible proportion, en Europe, de jeunes diplômés du supérieur. En 2011, ils étaient 20,3 \% en Italie, contre 43,4 \% en France. 
employeur. L'ISFOL souligne le fait que, pour la période 2007-2008, la majeure partie des ruptures de contrats d'apprentissage intervient avant la fin de la période de formation prévue. Dans un cas sur deux (48\%), les ruptures ont lieu au cours des trois premiers mois (Facello, 2013, p. 28). Les données les plus récentes confirment que ce sont toujours plus d'un tiers des contrats d'apprentissage signés qui sont rompus et dans la majorité des cas, suite à une démission de l'apprenti (INPS et INAPP, op. cit., p. 24-25).

Du point de vue de leur activité en entreprise, les apprentis se trouvent principalement dans quatre grands secteurs : l'industrie manufacturière de transformation (27\%), le commerce $(24 \%)$, la construction $(15 \%)$ et les services $(11 \%)$. La majorité des contrats d'apprentissage $(52 \%)$ est signée dans des entreprises de moins de 10 salariés ; la présence d'apprentis est inversement proportionnelle à la taille des entreprises.

Au regard des statistiques les plus récentes (INPS et INAPP, op. cit.), le portrait type de l'apprenti italien n'a guère évolué : il s'agit toujours d'un jeune homme (la proportion de femmes a baissé en 2016), âgé d'un peu moins de 25 ans (les apprentis étant plus âgés d'un an en moyenne dans le centre et le sud de l'Italie), et engagé dans un apprentissage de type 2 (professionalizzante) dans une petite entreprise du nord du pays (généralement de moins de dix salariés) du secteur de l'industrie ou du commerce.

\section{L'inertie du fait apprenti en Italie}

Après avoir évoqué les limites de la réforme Fornero, décelables du point de vue statistique, on situe l'inertie du fait apprenti dans la diversité des pratiques d'engagement (2.1), des économies régionales (2.2) et plus globalement au sein de la situation socio-économique de l'Italie contemporaine (2.3).

\subsection{La concurrence des formes d'engagement}

En Italie, la diminution du nombre d'apprentis traduit la concurrence d'autres dispositifs conventionnels, notamment le "stage formateur" (tirocinio formativo). D'une durée de douze mois, ce dispositif vise à acquérir une expérience à travers une alternance entre études et travail, similaire à l'apprentissage. La réforme Fornero a voulu rigidifier ce type de stage ${ }^{26}$, pour lequel le versement d'une "indemnité adéquate " (congrua indennità) devenait obligatoire ${ }^{27}$, afin de favoriser l'apprentissage (Facello, 2013). Pourtant, parce qu'il ne prévoit aucun contrôle de la qualité et du contenu de la formation, les

26. Entre autres types de dispositifs contractuels.

27. Cette indemnité, versée par l'employeur, est d'environ 300 euros par mois. 
employeurs peuvent préférer le stage à l'apprentissage. La pratique des entreprises fait ainsi peser le risque que le stage ne devienne "une sorte de mini-job à l'allemande, avec encore moins de garanties" (Bertagna \& al., 2013, p. 417). Autrement dit, une forme potentiellement diffusée d'infra-emploi, très faiblement encadrée et rémunérée.

Plus récemment, le contrat "à protections croissantes ", introduit avec le "Jobs Act " de $2015^{28}$, est venu concurrencer le stage, en instaurant une exonération totale de cotisations patronales sur trois ans (jusqu'à un plafond annuel de 8060 euros) pour toute nouvelle embauche. Il a connu un essor immédiat, qui contribue à expliquer la baisse importante $(21 \%)$ de nouvelles embauches d'apprentis en 2015 (INPS et INAPP, op. cit., p. 17). En revanche, en 2016, le nombre de nouveaux contrats d'apprentissage signés a augmenté de $32 \%$ par rapport à 2015, suite à la réduction des incitations à l'embauche en contrat "à protections croissantes"(ibid.). Ces variations confirment l'ancrage de l'embauche d'apprentis dans le rapport coût/bénéfice, au sein de l'offre de contrats disponibles en Italie.

Ce faisant, cette concurrence, portant sur la fonction " occupationnelle » de l'apprentissage, introduit une tension dans le dispositif. En effet, la particularité de l'apprentissage est de considérer l'emploi non comme une finalité, mais comme un moyen permettant de se former, ce qui implique de nuancer l'inscription de l'apprenti dans une logique de production pure. De ce point de vue, l'apprentissage se heurte à l'incertitude sur l'engagement des employeurs en matière de formation des apprentis, mais aussi à l'implication inégale des régions dans un suivi effectif de l'apprentissage, en lien avec une très grande disparité de moyens à disposition.

\subsection{Des disparités régionales...}

Les très fortes disparités socio-économiques entre les vingt régions italiennes se retrouvent également en matière d'apprentissage, où la Lombardie à elle seule concentre 17,8 \% de l'ensemble des contrats apprentis enregistrés au niveau national. En 2013, le responsable d'un Centro formativo provinciale (CFP), à Brescia (Lombardie), évoque ces contrastes régionaux en matière de financement, de services (conseils, accompagnement, etc.), de construction de cours adaptés, de recrutement de professeurs et de maillage territorial. Ces disparités s'ajoutent à celles existant entre professions (en matière de réglementation par exemple) et entreprises (en matière de politiques salariales, de gestion RH, etc.), qui déterminent de manière décisive la réussite ou l'échec des politiques de promotion de l'apprentissage (Muehlemann \& al., op. cit., p. 11). Ces inégalités se retrouvent ensuite en matière d'insertion professionnelle, puisque $60 \%$ des contrats d'apprentissage transformés en CDI (contrat à durée indéterminée) le sont dans le Nord.

28. Le "Jobs Act» désigne le décret loi du 23 mars 2015, promulgué par le gouvernement de Matteo Renzi (centre-gauche). 
Toutefois, même en Lombardie, région industrialisée où les apprentis sont en plus grand nombre, la mise en concurrence des dispositifs d'engagement est prégnante ${ }^{29}$. Suivant le responsable de CFP cité, cette pratique est générale dans les entreprises formatrices. Selon lui, si elles peuvent concevoir l'apprentissage comme un investissement, dans la majorité des cas, elles ne l'évaluent positivement qu'en lien avec les exonérations de cotisations sociales accordées.

Cette très forte sensibilité des employeurs aux incitations liées au contrat traduit le caractère secondaire de la dimension formation. C'est ainsi qu'à l'époque de la réforme Fornero, seuls $26 \%$ des apprentis ont participé à un cours de formation professionnelle agréé par la région (ou une province autonome). Mais ici aussi, les disparités sont fortes : tandis que $40 \%$ des apprentis du nord du pays sont insérés dans un parcours de formation externe à l'entreprise, ils sont moins de $10 \%$ dans les régions du centre et du sud (ISFOL, op. cit., p. 15). La reconduction, en 2012, des trois types d'apprentissage, introduite par la réforme Biagi, n’a pas modifié cette situation.

L'apprentissage donne lieu ainsi à des interprétations extensibles en matière d'âge, de rémunération, de formation, dans un brouillage qui rend difficile la validation des compétences acquises dans le cadre de la formation professionnelle en entreprise (Azaïs, 2009). Les nombreux cas de ruptures de contrat d'apprentissage de type 1 (16-18 ans), à l'initiative des entreprises, paraissent motivés par des périodes de cours jugées trop importantes. "Les entreprises veulent avant tout pouvoir disposer des apprentis sur le lieu de travail, afin quils remplissent leur charge de travail programmé", selon le responsable du Centro formativo provinciale (CFP) à Brescia, déjà cité. À titre d'illustration, ce dernier évoque dix-sept apprentissages ayant effectivement commencé en 2013, sur 300 prévus pour cette tranche d'âge, ou des ruptures de contrat survenues avec des apprentis non-majeurs, les employeurs attendant leur majorité pour signer un nouveau contrat, qui n'impose pas le suivi d'autant d'heures d'enseignement ${ }^{30}$.

Ces propos font écho aux constats du Cedefop, qui souligne le manque d'implication (coinvolgimento) des entreprises en faveur de l'apprentissage de type 1 et l'attribue non seulement à un manque général d'information et de connaissance du dispositif, mais aussi au non-respect de la part formative du contrat, qui ne se limite pas à envoyer l'apprenti dans un centre de formation, mais doit aussi le préparer à la qualification visée. À cette indistinction entre travail au sein de l'entreprise et formation, s'ajoute la difficulté d'établir un planning conciliant heures de travail en interne, d'un côté, et heures de formation en externe, de l'autre (Cedefop, op. cit, p. 19).

29. Rappelons qu'en Italie, un contrat d'apprentissage peut être signé jusqu'à l'âge de 29 ans.

30. Ne se contentant pas de relever les comportements des entreprises, ce responsable de formation rend également les jeunes, et parmi eux les mieux diplômés, pour partie responsables du fort taux de chômage qui les touche, en raison de leur attachement supposé trop grand au contrat à durée indéterminée. Il plaide ainsi en faveur de leur adaptation à l'emploi atypique, alternatif à l'emploi en CDI à temps plein. 


\section{3... À lier à une situation économique et sociale nationale}

Les disparités régionales concernant l'apprentissage doivent être corrélées avec la physionomie du système éducatif italien, mais aussi avec l'expérience de l'État italien dans le cadre des politiques publiques préconisées au niveau communautaire.

En Italie, la démocratisation scolaire est marquée par un fort taux d'abandon d'études, un faible accès à l'université et un faible investissement des entreprises dans la formation professionnelle continue. En ce sens, l'Italie présente un paysage de l'enseignement professionnel aux contours moins nets qu'en France (avec les filières professionnelles du baccalauréat au BTS - brevet de technicien supérieur -, voire au-delà) ou qu'en Suisse (avec le modèle dual). La construction d'un système homogène de standards professionnels, éducatifs et certifiants, auquel pourrait s'adosser l'ensemble des parcours de formation, constitue ainsi un enjeu important au sein du système éducatif italien (Forcello, 2013, p. 40). Dans cet environnement institutionnel, évoquant une "convention méritocratique " inaccomplie, c'est-à-dire manquant d'intégrer l'ensemble des jeunes dans une trajectoire éducative et professionnelle sanctionnée par un diplôme d'État (Verdier, 2001), l'apprentissage peine à trouver sa légitimité.

En outre, l'Italie connaît une crise économique aigüe et une pression européenne très forte pour diminuer les dépenses publiques et assouplir le fonctionnement du marché du travail. Dans ce contexte, la réforme Fornero de 2012 visait à faciliter l'accès ou le retour à l'emploi des jeunes en misant sur l'apprentissage. Or, cette réforme intervient dans un environnement contractuel complexe, l'un de nos interlocuteurs syndicaux n'hésitant pas à parler de "jungle ", encore accrue par le "Jobs Act " ${ }^{31}$. Dans ce cadre, l'échec de la réforme, sur le plan quantitatif, confirme l'arbitrage des entreprises, entre opportunités économiques et contraintes règlementaires, dans le choix d'un support contractuel pour l'embauche de jeunes.

31. En Italie, le lien d'emploi est codifié, depuis 1970, par le Statut du travailleur, véritable pivot des relations professionnelles et de travail. Après plusieurs tentatives limitées, le "Jobs Act " abroge l'article 18 $\mathrm{du}$ Statut, prévoyant la réintégration automatique du travailleur injustement licencié, et prédéfinit le montant de l'indemnité compensatoire en cas de licenciement irrégulier (ce qui inspirera, en France, la loi El Khomri, ou " loi Travail ", votée en août 2016). Comme preuve ultérieure d'un environnement juridique instable, des dispositions importantes du "Jobs Act » ont ensuite été mises en cause avec le vote du décret loi « Dignité » (Dignità), signé par le ministre du Travail L. Di Maio et entré en vigueur le 13 juillet 2018. Ce texte prévoit une série de mesures censées réduire la précarité juridique et financière des travailleurs qu’a impliquée le "Jobs Act». Il vise aussi à simplifier les procédures fiscales pour les entreprises, tout en pénalisant celles qui délocaliseraient après avoir bénéficié d'aides publiques. 


\section{Les usages situés 3 de l'apprentissage}

Après avoir contextualisé les pratiques de l'apprentissage en Italie, on envisage deux formes d'encastrement permettant d'expliquer son recours : les compromis au sein d'une entreprise familiale typique de la région de Brescia (Lombardie) (3.1) et la perspective d'émigration d'un jeune géomètre diplômé d'un institut technique (3.2).

\subsection{Embauches à bas coût et logiques familiales}

Au cours de notre enquête, nous avons pu observer comment, dans une entreprise familiale de commerce de moins de dix salariés, dans une petite ville de Lombardie, la signature d'un contrat d'apprentissage était systématique pour l'ensemble des salariés de moins de 29 ans. La justification avancée est alors simple : au moment de l'observation, c'est le type de contrat de travail qui permettait à l'entreprise de réduire au maximum ses coûts salariaux à court terme. Toutefois, au-delà de cette justification spontanée, ce cas permet aussi de mettre en évidence l'inscription du recours à l'apprentissage dans un contexte de vie.

Dans cette petite entreprise de commerce, typique de la région, le choix de l'apprentissage s'appréhende en relation à une logique familiale excédant le périmètre de la famille nucléaire (foyer parents-enfant(s)). Les propriétaires actuels de l'entreprise sont les trois enfants du fondateur de l'activité, débutée avant-guerre comme bazar de centre-ville. L’entreprise se compose aujourd'hui de deux activités séparées : d'une part, un magasin de jouets et de petits articles pour la maison, que dirigent les deux sœurs et qui emploie deux vendeuses ; d'autre part, un magasin de cuisines et d'ameublement, dirigé par le frère, et qui emploie le mari de l'une des deux sœurs, le fils de l'autre sœur, ainsi que trois ouvriers installateurs de cuisine. Ces deux magasins mitoyens sont situés au rez-de-chaussée d'un bâtiment, construit par le fondateur de l'activité au cours des années 1960. Les premier et second étages de l'immeuble sont occupés par des logements.

Ici, le recours à l'apprentissage concerne Andrea ${ }^{32}$, fils d'une des deux sœurs propriétaires. Après une licence de sociologie (laurea breve) et sans expérience professionnelle, Andrea est embauché comme apprenti vendeur, à l'âge de 24 ans, dans le magasin de cuisines et ameublement (commesso alla vendita al pubblico). C'est un apprendistato professionalizzante (type 2) de 48 mois, et de 40 heures hebdomadaires (dont 120 heures annuelles de formation en entreprise et en externe). Comme le reconnaît sans ambages l'un des propriétaires, le recours au contrat d'apprentissage était alors le plus «avantageux» (conveniente) : il «permettait de payer moins" et de "récupérer le plus en cotisations", à condition d'attester de sa présence aux cours dispensés hors de l'entreprise.

32. Prénom fictif. 
Compte tenu du contexte, l'embauche d'Andrea était d'emblée acquise à la fin de son contrat d'apprentissage, qui aura duré quatre ans, jusqu'en 2010, comme initialement prévu. Dans les faits, les activités exercées excèdent les compétences strictes de vendeur et incluent des dimensions plus techniques : maîtrise d'outils informatiques (dessin assisté par ordinateur), installation de cuisines, notions de comptabilité, etc. Cette variété d'activités est liée à la nature de l'entreprise, dont les effectifs sont réduits et le carnet de commandes soumis à des fluctuations. Dans ce contexte, le recours à l'apprentissage vise à tester les capacités de la personne dans un univers de production aux exigences polyvalentes. Par ailleurs, il s'agissait, dans l'esprit de tous, de trouver la formule contractuelle qui permettrait à l'entreprise de maximiser des profits ensuite socialisés au sein de la famille, donc y compris au bénéfice de ce même Andrea. En outre, ce dernier occupe un logement indépendant et gratuit, aux étages supérieurs du bâtiment, mitoyen de celui des parents, du grand-père fondateur et de la fille de l'une des sœurs propriétaires. D'autres dépenses, comme les repas du midi, pris ensemble, les frais d'eau, de gaz et d'électricité du logement individuel, sont encore pris en charge par l'entreprise et représentent autant de compléments à la rémunération directe de l'apprenti, qui ne peut donc être considérée comme source exclusive de revenus.

Ainsi, le contrat ne fait pas tout : son recours prend sens dans une situation, comme le montre la configuration entrepreneuriale et familiale décrite ici. Si les arrangements y sont singuliers, on peut envisager ce cas comme l'expression significative de l'apprendistato professionalizzante, le type d'apprentissage le plus répandu, dans une entreprise de moins de dix salariés, dans un contexte national où $90 \%$ des entreprises sont familiales et où les dirigeants appartiennent, plus qu'ailleurs, à la famille des initiateurs de l'activitéz ${ }^{33}$.

D'autres traits de l'expérience apprentie au sein de cette entreprise viennent donner corps à l'analyse statistique, touchant la difficile régulation de la formation. L'une des propriétaires nous explique ainsi que l'un des trois installateurs de cuisine a également été apprenti dans l'entreprise avant d'être embauché. À l'époque, le nombre insuffisant d'apprentis préparant ce métier ne permettait pas au CFP d'ouvrir des cours correspondants. À la place, l'apprenti a dû suivre des cours de comptabilité. En outre, suite au départ de deux de ses installateurs, l'entreprise familiale a recruté un nouvel employé en 2015. Cette embauche succède à un "stage formateur ", que des dispositions ultérieures à son introduction par la réforme Fornero, ont consacré comme un support de transition vers l'emploi. Ici encore, la dimension économique du dispositif a fait primer le recours au stage sur l'apprentissage en entreprise ${ }^{34}$.

33. Source : Osservatorio AUB (http://www.aidaf.it/attivita/studi-e-ricerche/). Tandis que les deux tiers des entreprises familiales italiennes n'ont pas recours à des managers externes à la famille des entrepreneurs, ce pourcentage est de $26 \%$ en France et de $10 \%$ au Royaume-Uni (http://www.aidaf.it/aidaf/le-aziende-familiari-in-italia/).

34. L'entreprise versait 600 euros d'indemnité mensuelle au stagiaire, alors qu'elle aurait dû lui verser un salaire plus important, autour de 900 euros plus une part variable selon les jours de présence en entreprise, 


\subsection{L'apprentissage en vue de l'expatriation}

De manière complémentaire à celle d'Andrea, l'expérience de Luca ${ }^{35}$, qu'il restitue dans un entretien mené en 2013, permet de mettre en lumière une autre perspective de formation et d'insertion professionnelles, cette fois en vue d'une expatriation.

Luca a une vingtaine d'années, il est né et a grandi dans la région napolitaine. Il est de double nationalité, italienne, et suisse par son père, qui l'a acquise après avoir travaillé de nombreuses années comme ouvrier en Suisse. Luca a choisi de devenir géomètre et a opté, parmi les différentes voies possibles, pour celle de la formation professionnelle dans un institut technique (istituto tecnico ${ }^{36}$ ). Cette dernière prévoyait deux années d'apprentissage "per la qualifica e per il diploma professionale " (type 1) auprès d'un professionnel déjà établi. Il lui fallait donc trouver un employeur susceptible de l'engager, afin de pouvoir se présenter à l'examen et obtenir un certificat de qualification professionnelle lui permettant d'exercer en Italie ou dans un autre pays de l'Union européenne, ainsi qu'en Suisse.

Luca signe son contrat au début des années 2010. Au cours de sa formation en entreprise, il n'a perçu aucune rémunération (même sous forme de remboursement de frais ou " au noir »), ni aucune aide pour préparer ses examens finaux. Il n'a exécuté qu'un ensemble de tâches directement utiles à l'activité de son employeur. L'échange, consistant à accepter la réalisation de tâches faiblement ou non rémunérées contre un apport de formation " en nature ", se trouvait ainsi dévoyé. Luca dénonce rétrospectivement la prestation d'un « travail gratuit » contre la possibilité de présenter l'examen.

De plus, son témoignage traduit ce que l'on pourrait qualifier de double inadéquation. D’un côté, l'activité sur le lieu d'exercice professionnel est perçue comme répétitive, peu valorisante, spécifique à l'employeur et sans lien direct avec les savoirs exigés à l'examen. Mais inversement, aux yeux de l'apprenti, les savoirs et compétences enseignés en école le sont sous une forme sans rapport avec la pratique professionnelle. Au désintérêt de l'employeur pour la formation s'ajoute le décalage perçu par Luca entre formation reçue en école et exercice professionnel.

Toutefois, la possession d'une double nationalité a permis à Luca d'élaborer, en compensation, une stratégie d'insertion. Il lui fallait " un bout de papier ", c'est-à-dire un diplôme en Italie, mais reconnu en Suisse, où il pourrait le faire valoir et concourir aux emplois vacants sur place. Alors qu'en France ou en Suisse, la perspective d'obtenir un revenu est un puissant facteur incitatif à choisir la voie de l'apprentissage, un tel moteur est absent dans le cas de ce jeune Italien. A contrario, sa stratégie met en évi-

en cas d'une embauche comme apprenti.

35. Prénom fictif.

36. Suite à la réforme dite de la "Buona scuola" (loi 107/2015), ce diplôme se prépare aujourd'hui dans un Istituto Tecnico settore tecnologico ad indirizzo Costruzioni, Ambiente e Territorio (CAT). 
dence un fait aujourd'hui largement connu en Italie : la fuite des jeunes à l'étranger, et notamment des jeunes diplômés. ${ }^{37}$

Dans le cas de Luca, le contrat d'apprentissage s'inscrit dans le prolongement de sa fréquentation d'un institut technique. La pratique de l'employeur contredit cependant le fait de l'envisager comme un support de formation qualifiante et d'emploi pérenne. Par contraste avec le cas précédent, cette expérience d'apprenti nourrit une dénonciation récurrente en Italie : celle d'un recours à l'apprentissage, par les entreprises, pour verser un salaire plus faible que la normale. Cela justifie un appel au contrôle des règles régissant l'apprentissage sur le lieu de travail (horaires, niveau de salaire, contenu de l'activité, etc.), devant permettre de limiter le chantage à l'emploi ${ }^{38}$. Toutefois, Luca trouve une solution individuelle : il inscrit l'apprentissage dans une perspective d'émigration, largement à sa charge. L'horizon normatif de l'apprentissage, celui de former au plus près des réalités productives, cède ainsi le pas à un phénomène d'expatriation sélective $^{39}$, dans un contexte marqué par la crise entamée en 2008, qui contribue à étendre la précarité au sein d'une génération qui se qualifie elle-même de "génération 1000 euros" (Generazione Mille Euro) ${ }^{40}$. La crise doit aussi être considérée comme un facteur d'érosion de la fonction traditionnelle des familles, comme "amortisseur social» (ammortizzatore sociale), notamment dans le sud du pays, avec l'augmentation de la pauvreté (Istat, 2018).

\section{Conclusion : des encastrements multiniveaux jouant en défaveur du développement de l'apprentissage en Italie}

La corrélation entre le " coût " de l'apprentissage, comparativement à d'autres modalités contractuelles, et le non-décollage du nombre d'apprentis, est confirmée par notre enquête. Toutefois, nos données empiriques sur les usages du dispositif invitent à ne pas négliger l'encastrement social de ce relatif (non-)recours. Les deux cas étudiés invitent ainsi à considérer que la stagnation de l'apprentissage, en Italie, tient à la nature des organisations de travail, largement dominées par de petites entreprises.

37. Par exemple : "Esplode fuga da Italia, + 30 \% emigrati », Ansa, 7 avril 2013.

38. Voir par exemple l'article de presse intitulé « Nous, apprentis juste de nom : seulement un moyen pour moins nous payer" ("Noi, apprendisti solo di nome : cosi ci pagano meno »). Mattia Vito, apprenti (de type 2 ou professionalizzante) de 24 ans, y décrit sa situation : marié, percevant un salaire mensuel de 800 euros, plus faible que celui de ses collègues, alors qu'il accomplit rigoureusement le même travail quotidiennement.

39. L'expatriation s'appuie en effet sur des ressources inégalement distribuées : accès à l'information, capacité financière à assumer le voyage, " point de chute ", réseau sur place, détention d'un passeport du pays d'émigration, etc.

40. Peu avant la réforme Fornero, le rapport annuel de l'ISTAT, en 2011, établissait que plus d'un Italien de moins de 35 ans en emploi sur deux l'était sous le régime d'un contrat atypique et différent d'un CDI à temps plein (CDD, intérim, contrats de collaboration, contrats à projets, etc.). 
Dans ce contexte, le recours à l'apprentissage est évalué de manière concurrentielle aux autres possibilités contractuelles, non seulement du point de vue du coût, mais aussi de la souplesse de l'engagement, au sein d'une division du travail distincte de celle d'une grande entreprise. De plus, les deux cas rapportés ici font apparaître une situation différenciée de l'apprenti, selon que son apprentissage se déroule dans l'entreprise de sa propre famille ou non : à la chance d'obtenir un emploi pérenne dans un cas se substitue la dénonciation du travail gratuit dans l'autre.

$\mathrm{Au}$ vu de ces résultats, on comprend mieux l'échec de la réforme Fornero : ses ambitions réformatrices n'ont pas démêlé l'écheveau contractuel italien, ni les disparités régionales profondes qui affectent le pays. Celles-ci se traduisent par la marginalité du fait apprenti dans les régions les plus fragiles économiquement (centre et sud), mais aussi par l'hétérogénéité territoriale de l'offre publique de formation pour les apprentis et des différences entre employeurs dans leur rôle d'encadrant de la formation en entreprise. Les chiffres contemporains de l'apprentissage confirment cette analyse : le contrat à protections croissantes, introduit par le Jobs Act, n'a fait qu'alimenter la concurrence entre différents dispositifs incitatifs et s'est traduit par la baisse des effectifs apprentis depuis 2009, malgré une légère reprise en 2016 (INPS et INAPP, op. cit.).

Dès lors, on ne peut qu'être sceptique devant l'idée, avancée par certains, selon laquelle l'abaissement des salaires apprentis, par le creusement de l'écart ainsi créé avec le salaire du professionnel qualifié de référence, peut constituer un moyen d'accroître l'attractivité du dispositif (Muehlemann \& al., op. cit., p. 1141). En effet, si le coût du travail est fondamental dans le choix des employeurs, on a vu que ces derniers n'hésitent pas à prendre de franches libertés avec le volet formation de l'apprentissage. Abaisser à nouveau le niveau de rémunération (déjà diminué en 2015) du contrat d'apprentissage, sans améliorer le volet formation, achèverait d'en dévoyer le volet emploi, dans le contexte d'une austérité salariale expérimentée à l'échelle nationale ${ }^{42}$.

Enfin, on peut mettre le cas italien en perspective avec la situation française, où, en écho aux incitations européennes (Commission européenne, 2012, 2013), l'apprentissage est aussi promu par les pouvoirs publics. Les difficultés et succès rencontrés pour augmenter les effectifs apprentis demeurent largement spécifiques à chaque configuration nationale. Néanmoins, dans les deux cas, le sens conféré à l'apprentissage par les promoteurs politiques et patronaux relève de la construction de l'employabilité dès le début du parcours professionnel, par un processus d'acquisition de compétences "piloté par l'aval» (Stroobants, 2007). Pourtant, son institutionnalisation postulée ne

41. D’après les auteurs, le salaire apprenti ne doit pas dépasser $50 \%$ du salaire du professionnel qualifié pour que l'apprentissage présente un bilan coûts-avantages positif.

42.Voir par exemple : https://ec.europa.eu/eurostat/statistics-explained/index.php?title=Wages_and_labour_costs/it 
semble pas modifier le fait que l'apprentissage est considéré comme un salariat à bas coût par les employeurs italiens - pratique qu'il conviendrait d'interroger en France, où les employeurs disposent aussi d'une pluralité de possibilités contractuelles, notamment en alternance (Arrighi \& Mora, 2010).

\section{Bibliographie}

Azaïs C. (2009), « Les enjeux de la relation formation-emploi en Italie: la place du travail et des territoires ", Espaces et Sociétés, n 136-137, pp. 63-77.

Arrighi J.-J. \& Mora V. (2010), «Apprentissage contre professionnalisation : un faux débat ", Bref-Céreq, n 276.

Bertagna G., Buratti U., Fazio F. \& Tiraboschi M. (dir.) (2013), « La regolazione dei tirocini formativi in Italia dopo la legge Fornero, Adapt ", e-book series, n 16 (en ligne).

Bobba L. (2015), "Il rilancio dell'apprendistato per un sistema duale italiano », Osservatorio Isfol, V, n. 3, pp. 13-18.

Cedefop (2017), Analisi tematica sull'apprendistato in ITALIA Creare opportunità d'istruzione e formazione attraverso l'apprendistato, Luxembourg, Ufficio delle pubblicazioni. Analisi tematiche per paese.

Cefalo R. (2015), Alla ricerca di una cultura dell'apprendistato in Italia, Secondowelfare.it (en ligne).

Commission européenne (2012), The effectiveness and costs-benefits of apprenticeships: Results of the quantitative analysis, Bruxelles.

Commission européenne (2013), Apprenticeship and Traineeship Schemes in EU27: Key Success Factors, Bruxelles.

Confcommercio (2014), Rapporto annuale sul mercato del lavoro: l'apprendistato (en ligne).

Dif-Pradalier M. \& Zarka S. (2014), Redonner ses chances à l'apprentissage. Une comparaison France, Suisse et Italie, Paris, CFTC-IRES.

Facello S. (2013), Apprendistato e tirocini :il ruolo dell'alternanza, thèse de doctorat en droit du travail, Università degli studi di Bergamo.

Granato M. \& Moreau G. (2019), «Introduction », Formation Emploi, n 146, pp. 7-28 (en ligne).

ISFOL (2011), XI Rapporto di monitoraggio sull'apprendistato, pubblicato dal Ministero del Lavoro e realizzato con il contributo dell'Isfol (en ligne).

ISFOL (2012), XIII Rapporto di monitoraggio sull'apprendistato, pubblicato dal Ministero del Lavoro e realizzato con il contributo dell'Isfol (en ligne). 
INPS \& INAPP (2017), Verso una ripresa dell'apprendistato. XVII Rapporto di monitoraggio (en ligne).

INAPP (2017), « Dati inediti da Rapporto Apprendistato 2017 », Comunicato stampa (en ligne).

ISTAT (2018), Rapporto annuale 2018. La situazione del Paese (en ligne).

Muehlemann S., Wolter S. C. \& Joho E. (2018), Apprenticeship training in Italy. A costeffective model for firms?, Gütersloh, Bertelsmann Stiftung (en ligne).

OECD (2017a), OECD Skills Strategy Diagnostic Report. Italy, Paris, OECD.

OECD (2017b), Getting Skills Right. Italy, Paris, OECD.

Pastore F. (2014), «Il difficile percorso dell'apprendistato », Lavoce.info (en ligne).

Stroobants M. (2007), "La fabrication des compétences, un processus piloté par l'aval ?", Formation Emploi, n 99, pp. 89-94 (en ligne).

Teoldi F., Garibaldi P. (2012), La riforma è fatta. Lunga vita alla prossima riforma, lavoce. info (en ligne).

Tiraboschi M. (2015), Prima lettura del d.lgs. n. 81/2015 recante la disciplina organica dei contratti di lavoro, Bergamo, Adapt University Press.

Verdier E. (2001), "La France a-t-elle changé de régime d'éducation et de formation ? ", Formation Emploi, $\mathrm{n}^{\circ} 76$, pp. 11-24.

Wolter S. C. \& Schweri J. (2002), « The Cost and Benefit of Apprenticeship Training: The Swiss Case ", Applied Economics Quarterly (Konjunkturpolitik), n 48, vol. 3-4, pp. 347-367. 